\section{Parasite Burden and Severity of Malaria in Tanzanian Children}

\author{
Bronner P. Gonçalves, M.D., Chiung-Yu Huang, Ph.D., Robert Morrison, M.Sc., \\ Sarah Holte, Ph.D., Edward Kabyemela, M.D., Ph.D., D. Rebecca Prevots, Ph.D., \\ Michal Fried, Ph.D., and Patrick E. Duffy, M.D.
}

A BSTRACT

\section{BACKGROUND}

Severe Plasmodium falciparum malaria is a major cause of death in children. The contribution of the parasite burden to the pathogenesis of severe malaria has been controversial.

\section{METHODS}

We documented P. falciparum infection and disease in Tanzanian children followed from birth for an average of 2 years and for as long as 4 years.

\section{RESULTS}

Of the 882 children in our study, 102 had severe malaria, but only 3 had more than two episodes. More than half of first episodes of severe malaria occurred after a second infection. Although parasite levels were higher on average when children had severe rather than mild disease, most children (67 of 102) had high-density infection (>2500 parasites per 200 white cells) with only mild symptoms before severe malaria, after severe malaria, or both. The incidence of severe malaria decreased considerably after infancy, whereas the incidence of high-density infection was similar among all age groups. Infections before and after episodes of severe malaria were associated with similar parasite densities. Nonuse of bed nets, placental malaria at the time of a woman's second or subsequent delivery, high-transmission season, and absence of the sickle cell trait increased severe-malaria risk and parasite density during infections.

\section{CONCLUSIONS}

Resistance to severe malaria was not acquired after one or two mild infections. Although the parasite burden was higher on average during episodes of severe malaria, a high parasite burden was often insufficient to cause severe malaria even in children who later were susceptible. The diverging rates of severe disease and highdensity infection after infancy, as well as the similar parasite burdens before and after severe malaria, indicate that naturally acquired resistance to severe malaria is not explained by improved control of parasite density. (Funded by the National Institute of Allergy and Infectious Diseases and others.)
From the Laboratory of Malaria Immunology and Vaccinology (B.P.G., M.F., P.E.D.), Laboratory of Clinical Infectious DiseasesEpidemiology Unit (B.P.G., D.R.P.), and Biostatistics Research Branch (C.-Y.H.), National Institute of Allergy and Infectious Diseases (NIAID), National Institutes of Health (NIH), Rockville, MD; the Seattle Biomedical Research Institute (R.M., M.F., P.E.D.) and the Fred Hutchinson Cancer Research Center (S.H.) — both in Seattle; and the Mother-Offspring Malaria Studies Project, Muheza Designated District Hospital, Muheza, Tanzania (E.K., M.F., P.E.D.). Address reprint requests to Dr. Duffy at the Laboratory of Malaria Immunology and Vaccinology, NIAID, NIH, Twinbrook I, Rm. 1111, 5640 Fishers Lane, Rockville, MD 20852, or at patrick.duffy@nih.gov.

N Engl J Med 2014;370:1799-808. DOI: 10.1056/NEJMoal303944

Copyright (๑) 2014 Massachusetts Medical Society. 
LTHOUGH ALMOST 600,000 AFRICAN children die each year from malaria, ${ }^{1}$ most infections in children are mild. ${ }^{2,3}$ Fundamental questions about the pathogenesis of malaria remain unresolved, such as the relative contributions of parasite burden and host inflammation to severe disease. ${ }^{4}$ In areas where transmission is stable, severe malaria is unlikely to occur after 5 years of age, presumably as a result of immunity, ${ }^{5}$ and mathematical models suggest that protection against noncerebral severe malaria develops after one or two infections. ${ }^{6}$ The mechanism of protective immunity is unclear; it might, for example, involve the reduction of parasite density or the blocking of parasite virulence to prevent disease. IgG transferred from immune adults clears blood-stage parasites and symptoms in sick children, ${ }^{7}$ but the targets of protective IgG remain undefined.

To better understand the pathogenesis of severe malaria and acquired immunity, we undertook an intensive birth-cohort study of 882 children in northeastern Tanzania. We examined the relationship between the Plasmodium falciparum parasite burden and the severity of malaria within individual children over time and the risk of severe malaria during the first and subsequent infections.

\section{METHODS}

\section{STUDY POPULATION}

The study population was part of a longitudinal birth cohort in the Muheza district, an area of intense malaria transmission with an entomologic inoculation rate of approximately 400 infective mosquito bites annually. ${ }^{8}$ The incidence of malaria declined sharply in this area after the study closed in 2006. ${ }^{9}$ Newborns were enrolled in the study between September 2002 and November 2005. Children were followed for an average of 2 years and for as long as 4 years. Written informed consent was obtained from all the children's mothers before enrollment.

\section{STUDY PROCEDURES}

Children were examined at birth, once every 2 weeks during infancy, once every month after infancy, and during any illness. Blood smears were collected at all visits, regardless of whether symptoms were present. Parasitemia was defined as any P. falciparum detected in a Giemsa-stained blood smear, and high-density infection requiring parenteral treatment was defined as a parasite density of more than 2500 parasites per 200 white cells, in accordance with Tanzanian Ministry of Health and Social Welfare guidelines. P. falciparum-specific histidine-rich protein 2 (PfHRP-2) levels in plasma were measured with the use of a sandwich enzyme-linked immunosorbent assay (ELISA) ${ }^{10}$ (see the Supplementary Appendix, available with the full text of this article at NEJM.org).

Children were classified as having severe malaria in accordance with World Health Organization (WHO) criteria ${ }^{11}$ (hemoglobin level $<5$ g per deciliter, prostration, more than one convulsion in the past 24 hours, respiratory distress, or hypoglycemia). Children were classified as having moderately severe malaria if they did not fulfill the WHO criteria but had at least one of the following signs: a hemoglobin level lower than $6 \mathrm{~g}$ per deciliter, hyperthermia (temperature, $>40^{\circ} \mathrm{C}$ ), one convulsion in the past 24 hours, or a respiratory rate greater than 40 breaths per minute. Most cases of severe and moderately severe malaria (78.4\%) were treated with quinine. The results of analyses of moderately severe malaria are presented in the Supplementary Appendix. Children were classified as having mild high-density infection if they had a parasite density of more than 2500 parasites per 200 white cells in the absence of severe or moderately severe symptoms. Children were followed until the end of the study (May 2006) or until they dropped out of the study.

The study protocols were approved by the Division of Microbiology and Infectious Diseases at the U.S. National Institutes of Health and by the institutional review boards of Seattle BioMed and the Medical Research Coordinating Committee in Tanzania.

\section{STATISTICAL ANALYSIS}

All cases of parasitemia occurring within 28 days after a previous case were considered to be a single infection. We estimated age-specific incidence rates of infection, mild high-density infection, and severe malaria using a Nelson-Aalen estimator ${ }^{12}$ with confidence intervals calculated by means of a nonparametric bootstrap method. A generalized-estimating-equation approach for Poisson regression was used to compare agespecific rates of severe malaria and high-density infection. The risk of severe malaria during a 
first infection was estimated by dividing the number of children with severe malaria during a first infection by the total number infected at least once; similar risk calculations were performed for subsequent infections, after children with previous episodes of severe malaria were excluded. Log-transformed parasite density before and after a first episode of severe malaria was compared with the use of a paired t-test.

Cox proportional-hazards models were fit to determine the influence of study variables (village of residence, use or nonuse of bed nets, presence or absence of sickle cell trait, $\alpha$-thalassemia genotype, transmission season [low or high], birth season, sex, and a single variable combining status with respect to placental malaria and maternal parity) on time to first episode of severe malaria. A multivariate generalized-estimatingequation model with log-transformed parasite density as the dependent variable was developed to assess the effect of all study variables on the parasite burden. All reported P values are twosided; P values lower than 0.05 were considered to indicate statistical significance. Details of the survival analysis and generalized-estimatingequation methods are provided in the Supplementary Appendix.

\section{RESULTS}

\section{STUDY POPULATION}

During the recruitment period, 1045 mothers (of 1075 children) gave consent to participate and gave birth at the Muheza Designated District Hospital. After exclusions (see the Supplementary Appendix), 882 singleton infants were included in the analyses (Table 1), of whom 688 (78.0\%) were followed for at least 1 year. Baseline characteristics were similar in the overall population and among the children followed for more than 1 year. Most of the children (62.7\%) slept with bed nets, and among these children, 29.7\% slept with bed nets that were insecticide-treated. The prevalences of sickle cell trait (16.5\%) and $\alpha$-thalassemia (11.8\% homozygous and $40.9 \%$ heterozygous) were similar to those in other East African populations. ${ }^{13,14}$

The study encompassed 1762.8 child-years and 38,261 blood smears, including 6319 P. falciparumpositive blood smears representing 3933 independent infections; $65.4 \%$ of the positive blood smears were documented during scheduled vis-

\begin{tabular}{|c|c|}
\hline Characteristic & $\begin{array}{c}\text { Value } \\
(\mathrm{N}=882)\end{array}$ \\
\hline Maternal age $-\mathrm{yr}$ & $26.0 \pm 6.3$ \\
\hline \multicolumn{2}{|l|}{ Maternal parity - no. (\%) } \\
\hline First delivery & $254(28.8)$ \\
\hline Second delivery & $201(22.8)$ \\
\hline Third or subsequent delivery & $427(48.4)$ \\
\hline \multicolumn{2}{|l|}{ Maternal residence area — no. (\%) } \\
\hline Magila & $144(16.3)$ \\
\hline Muheza township & $400(45.4)$ \\
\hline Mkanyageni & $188(21.3)$ \\
\hline Bwembwera & $150(17.0)$ \\
\hline Bed-net use - no./total no. (\%) & $455 / 726(62.7)$ \\
\hline Birth weight — kg & $3.2 \pm 0.4$ \\
\hline Duration of follow-up - wk & $104.2 \pm 53.5$ \\
\hline \multicolumn{2}{|l|}{ Sex-no. (\%) } \\
\hline Male & $457(51.8)$ \\
\hline Female & $425(48.2)$ \\
\hline \multicolumn{2}{|l|}{ Transmission season at birth — no. (\%) } \\
\hline High & $432(49.0)$ \\
\hline Low & $450(51.0)$ \\
\hline Sickle cell trait — no./total no. (\%) & $142 / 861(16.5)$ \\
\hline Parasitemia during follow-up - no. (\%) & $715(81.1)$ \\
\hline Postneonatal death - no. (\%) & $35(4.0)$ \\
\hline Parasite-positive blood smears — no./total no. (\%) & $6319 / 38,261(16.5)$ \\
\hline \multicolumn{2}{|l|}{ Treated at home before presentation — \% } \\
\hline Severe malaria & 4.1 \\
\hline Mild malaria with high parasite density & 2.7 \\
\hline Mild malaria with any parasite density & 1.5 \\
\hline
\end{tabular}

* Plus-minus values are means \pm SD.

its. Of the 882 children, 715 (81.1\%) had at least one P. falciparum infection. A total of 102 children (11.6\%) had severe malaria, with 122 episodes of severe malaria overall. Most of the children who had severe malaria (99 of 102) had only one or two episodes. Of the 15 children who had a second episode, 7 presented with the same syndrome on both occasions, and 7 of 15 second episodes occurred during infancy (Fig. S6 in the Supplementary Appendix), when the incidence of severe malaria peaks. Of the 688 children followed for at least 1 year, 624 (90.7\%) became infected, and 98 (14.2\%) had severe malaria. 
Thirty-five children (4.0\%) died; 11 deaths were attributed to malaria. The overall mortality rate in our cohort was lower than that previously reported in this area ${ }^{15}$; the difference may be related to the intensive follow-up in our study (see the Supplementary Appendix). The proportion of childhood deaths attributable to malaria (31\%) was similar to that in other African communities. ${ }^{16-18}$

\section{PARASITE BURDEN AND SEVERE MALARIA}

The ranges of parasite densities during severe malaria and during mild or asymptomatic malaria overlapped considerably, both in the overall cohort (Fig. S1A in the Supplementary Appendix) and in the subset of children who had severe malaria during follow-up (Fig. S1B in the Supplementary Appendix). Assessment of PfHRP-2 levels, another measure of parasite biomass, had similar results (Fig. S1C in the Supplementary Appendix). The mean parasite density was substantially higher during episodes of severe malaria than during mild or asymptomatic infections $(\mathrm{P}<0.001)$, largely because severe malaria was rare during low-density infections (Fig. S2 in the Supplementary Appendix). However, most high-density infections were not associated with severe malaria: 253 of 882 children had a total of 444 highdensity infections with only mild symptoms, including 36 completely asymptomatic episodes.

Even among the 102 children who had severe malaria, most (67) had high-density infection with only mild symptoms before the first episode of severe malaria (21 children) (Fig. 1A), after the first severe episode (55 children) (Fig. 1B), or both. Both parasite density and PfHRP-2 levels were significantly higher during a mild high-density infection than during an episode of severe malaria; the mean ratio of parasite level during mild infection to parasite level during severe episodes was 6.3 (95\% confidence interval [CI], 3.8 to 10.5 ; $\mathrm{P}<0.001 ; 67$ children), and the geometric mean PfHRP-2 level was 4826 ng per milliliter $(95 \% \mathrm{CI}$, 3378 to 6894 ) during a mild high-density infection versus $1305 \mathrm{ng}$ per milliliter (95\% CI, 516 to 3298) during a severe episode ( $\mathrm{P}=0.01 ; 39$ children).

\section{RISK OF FIRST EPISODE OF SEVERE MALARIA}

The incidence of infection increased during the first 6 months of life, then remained stable (Fig. 2A). The change in timing of the analysis of blood smears from every 2 weeks to every month might have resulted in an underestimation of the inci- dence of infection after infancy, but it would not have affected the persistent plateau thereafter. The overall cumulative incidence rates of infection, severe malaria, and mild high-density infection were, respectively, 2.41 (95\% CI, 2.21 to 2.62), 0.05 (95\% CI, 0.04 to 0.06 ), and 0.26 (95\% CI, 0.22 to 0.30 ) episodes per child-year. The incidence of severe malaria - but not the incidence of mild high-density infection - diminished after infancy (Fig. 2B). The incidence rates of infection and severe malaria are similar to those that have been reported in other areas of high transmission ${ }^{19,20}$ (see the Supplementary Appendix).

The median age at the first episode of severe malaria was 38.7 weeks (interquartile range, 28.6 to 63.8). The risk of severe malaria did not decrease after the first infection; approximately $50 \%$ of children had at least two mild or asymptomatic infections before their first severe episode (Fig. 2C).

\section{SEVERE MALARIAL ANEMIA}

As in other African communities, ${ }^{21,22}$ most children with severe malaria typically presented with a single condition (Fig. 3), such as respiratory distress, convulsions, or severe anemia. Children had more than one condition in only $12.3 \%$ of severe episodes (15 of 122), which is similar to the proportions reported in previous studies. ${ }^{21,22}$ Children with convulsions were older and had lower parasite densities than children with severe anemia, although the differences were not significant ( $\mathrm{P}=0.09$ and $\mathrm{P}=0.21$, respectively) (Table $\mathrm{S} 1$ in the Supplementary Appendix). Of the 102 children who had severe illness, 6 (5.9\%) died during an episode of severe malaria. Three of the 15 children $(20 \%)$ who presented with overlapping severe malaria conditions died.

Most children with severe malarial anemia (26 of 40, 65\%) had their hemoglobin level measured in the previous 2 months. These children had a median reduction of $47 \%$ (interquartile range, 28 to 65 ) in the hemoglobin level at the time of severe malarial anemia. In 15 of the 26 children (58\%), hemoglobin levels were higher than $8 \mathrm{~g}$ per deciliter in the 2 months before the episode of severe malarial anemia, which suggests that there was a decrease of more than $3 \mathrm{~g}$ per deciliter during the acute infection. Fewer children (8 of 26, 31\%) had a hemoglobin level lower than $7 \mathrm{~g}$ per deciliter in the 2 months before an episode of severe malarial anemia. 
Figure 1. Risk of Severe Malaria and Parasite Density. Panel A shows high-density infections with only mild symptoms (blue circles) that occurred before severe malaria episodes (red circles) in 21 of 102 children, shown according to parasite density and age. Parasite levels during mild high-density infections that occurred before an episode of severe malaria were higher on average than parasite levels during episodes of severe malaria (mean ratio, 8.0; 95\% confidence interval [Cl], 3.0 to 21.2 ). The median time from the episode of high-density infection to the first episode of severe malaria was 154 days (interquartile range, 105 to 222). Panel B shows high-density infections with only mild symptoms that occurred after severe malaria episodes in 55 of 102 children, shown according to parasite density and age. Parasite levels during mild high-density infections after severe malaria were higher than parasite levels during episodes of severe malaria (mean ratio, 5.0; $95 \% \mathrm{Cl}, 2.9$ to 8.6 ). Panel $\mathrm{C}$ shows the mean parasite density of infections in the 9 months before, during, and in the 9 months after a child's first severe malaria episode. Only children who had at least one episode of parasitemia during the 9 months before and at least one episode of parasitemia during the 9 months after their first episode of severe malaria are included (58 children). The bar within each box indicates the median parasite density, the lower and upper ends of the box indicate the interquartile range, the bars below and above the boxes indicate 5 th and 95 th percentiles, and the circles indicate outliers.

\section{PLACENTAL MALARIA AND OTHER RISK FACTORS}

In Cox regression analyses of the time to the first episode of severe malaria, placental malaria and maternal parity were combined as one variable, because these factors interact to influence the incidence of infection. ${ }^{23}$ Bed-net use $(\mathrm{P}<0.001)$ and residence in Muheza or Mkanyageni $(\mathrm{P}<0.001$ and $\mathrm{P}=0.02$, respectively) reduced the risk of severe malaria, whereas the high-transmission season $(\mathrm{P}=0.05)$ and placental malaria at the time of second or subsequent delivery $(\mathrm{P}=0.04)$ increased the risk. In a multivariate Cox model (Table 2), bed-net use and residency in Muheza or Mkanyageni reduced the risk of severe malaria by $52 \%$, 61\%, and 53\%, respectively $(\mathrm{P}=0.001$, $\mathrm{P}<0.001$, and $\mathrm{P}=0.02$, respectively); sickle cell trait reduced the risk by $41 \%(\mathrm{P}=0.11)$, a finding that was similar to the results of previous studies. ${ }^{24}$ The high-transmission season increased the risk of severe malaria $(\mathrm{P}=0.05)$.

We fit a generalized-estimating-equation model with log-transformed parasite density as the dependent variable (Table 2). Sickle cell trait and bed-net use significantly reduced parasite density $(\mathrm{P}<0.001)$, whereas placental malaria at the time of second or subsequent delivery $(\mathrm{P}=0.04)$ and the high-

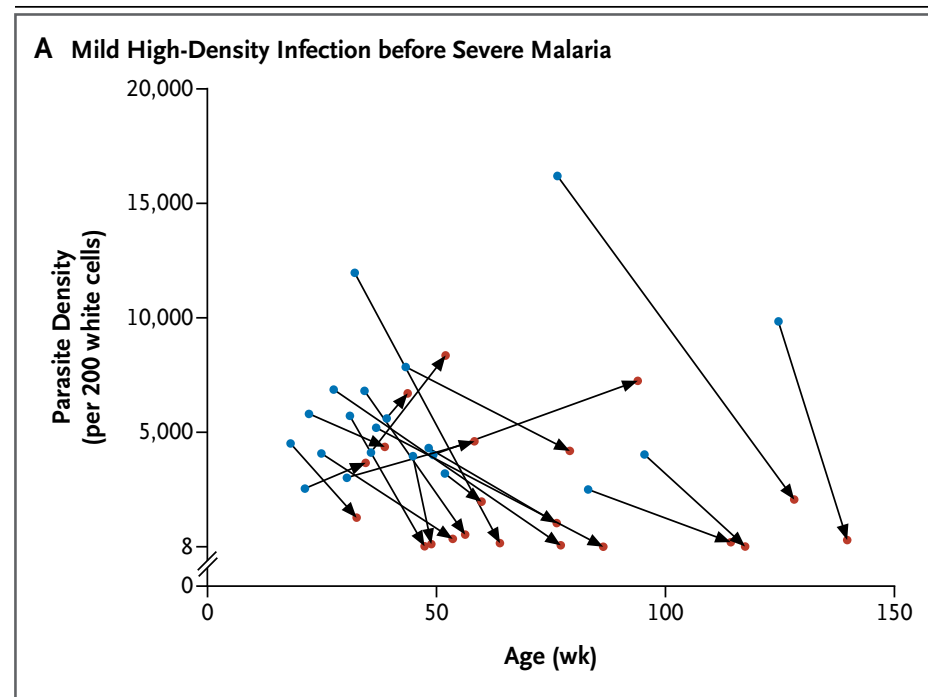

B Mild High-Density Infection after Severe Malaria

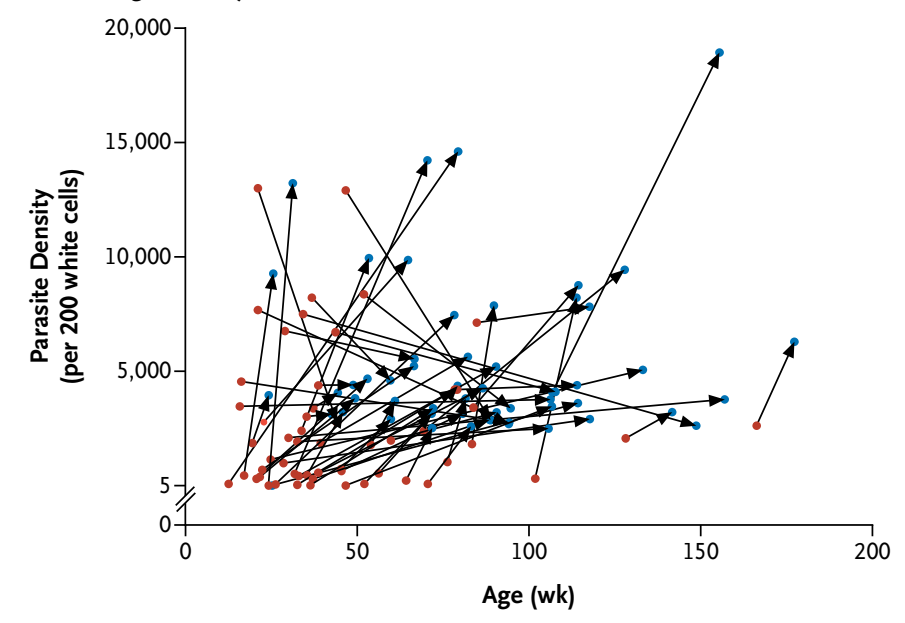

C Parasite Density before, during, and after Severe Malaria

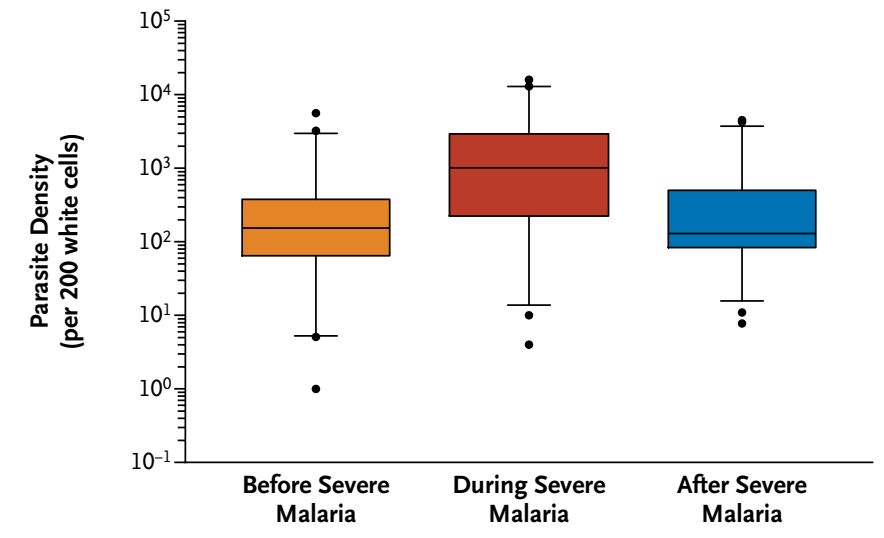

transmission season $(\mathrm{P}<0.001)$ significantly increased parasite density. The effects of these factors on parasite density parallel their effects on the risk of severe malaria, which suggests 


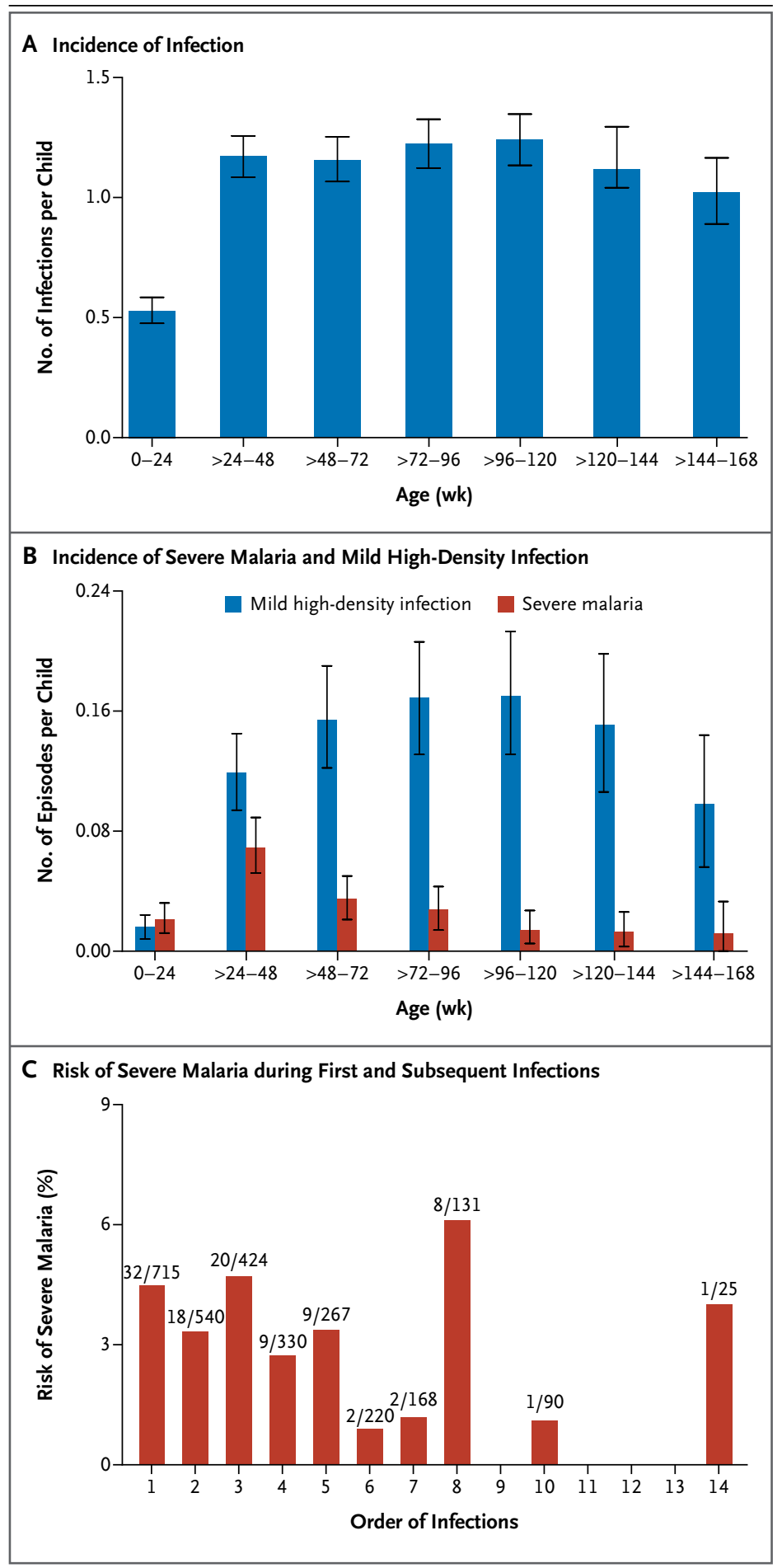

that interventions that reduce parasite density might prevent life-threatening disease by a mechanism that differs from naturally acquired resistance.
Figure 2. Risk of Severe Malaria, Infection, and HighDensity Infection.

Panel A shows the cumulative age-specific incidence rate of infections. Panel B shows the cumulative agespecific incidence rate of severe malaria and of highdensity infection with no more than mild symptoms. I bars in both panels indicate $95 \%$ confidence intervals. Panel $C$ shows the risk of severe malaria during sequential infections. In the fractions above each bar, the numerator is the number of children with severe malaria at that particular infection, and the denominator is the total number of children who had the indicated number of infections. For example, only 25 children had at least 14 infections, and 1 of those children had a first severe malaria episode at the time of that 14th infection.

\section{IMMUNITY TO SEVERE MALARIA AFTER INFANCY}

Rates of high-density infection and severe malaria (Table S3 in the Supplementary Appendix) did not differ significantly during the first 24 weeks of life (rate ratio, 1.61; $\mathrm{P}=0.07$ ), but rates of highdensity infection significantly exceeded rates of severe malaria thereafter (rate ratios of 2.26, 5.58, and 13.58 for children 24 to $48,>48$ to 96 , and $>96$ weeks of age, respectively; $\mathrm{P}<0.001$ for all comparisons) (Fig. 2B). Changes in the rates of high-density infection and severe malaria also diverged with age (Table S4 in the Supplementary Appendix): in comparison with peak incidence among children older than 24 weeks through 48 weeks of age, rate ratios for severe malaria were estimated as $0.46(\mathrm{P}<0.001)$ for children older than 48 weeks through 96 weeks of age and $0.16(\mathrm{P}<0.001)$ for children older than 96 weeks of age, which is consistent with acquired immunity. In contrast, the rate of high-density infection remained stable after 24 weeks of age.

Parasite densities in individual children did not differ significantly between infections that occurred in the 9 months before and those that occurred in the 9 months after the first episode of severe malaria ( 58 children, $\mathrm{P}=0.56$ ) (Fig. $1 \mathrm{C}$ ); infections during the first 4 months of life were excluded from this analysis, because parasite levels at this age are lower (Fig. S5 in the Supplementary Appendix). Assessment of PfHRP-2 levels had similar results (34 children; geometric mean level, 496 ng per milliliter [95\% CI, 245 to 1006] before vs. 717 ng per milliliter [95\% CI, 348 to 1477] after severe malaria; $P=0.30$ ). Similar results were also obtained with the use of a 3-month window rather than a 9-month window. 


\section{DISCUSSION}

Most of the children in our study had severe malaria after one or more previous infections, rather than during their first infection, and most such children had much higher parasite densities (six times as high) with only mild symptoms in previous or subsequent infections. Taken together, the data suggest that severe malaria cannot be explained by parasite burden alone and that factors such as parasite virulence and host inflammation might play key roles in immunopathogenesis. Furthermore, acquired immunity to severe malaria is not explained by improved control of parasite density and therefore might target distinct antigens or processes involved in severe syndromes.

On the basis of rates of infection and hospitalization due to malaria, the risk of noncerebral severe malaria was thought to be limited to the first infection or two. ${ }^{6}$ This prospective study provides evidence that the risk of severe malaria is stable over several infections..$^{25}$ As transmission decreases in many parts of Africa, the risk of severe malaria shifts to older age groups ${ }^{26}$ and the idea that only minimal exposure is required to generate immunity may lead to complacency regarding this danger. Conversely, the relatively poor efficacy of the antimalarial agents recommended at the time of our study (treatment with artemisinins was implemented as policy after our study ended) may have contributed to high transmission, as well as to the early peak incidence and rapid acquisition of immunity to severe malaria.

The low risk of recurrent severe malaria and the rapid decrease in the incidence of severe malaria after infancy are consistent with earlier evidence that immunity to severe malaria is acquired rapidly. ${ }^{6}$ Recurrences of severe malaria are equally likely to be the same syndrome as the first episode or a different syndrome (Table S6 in the Supplementary Appendix). This fact and the observation that a child's single episode of severe malaria is manifested as a single defining syndrome suggest that immunity to severe malaria is not syndrome-specific and therefore that targets of protective immunity may be conserved among syndromes.

Our data suggest that the total parasite burden contributes to the pathogenesis of severe malaria, although this association does not prove

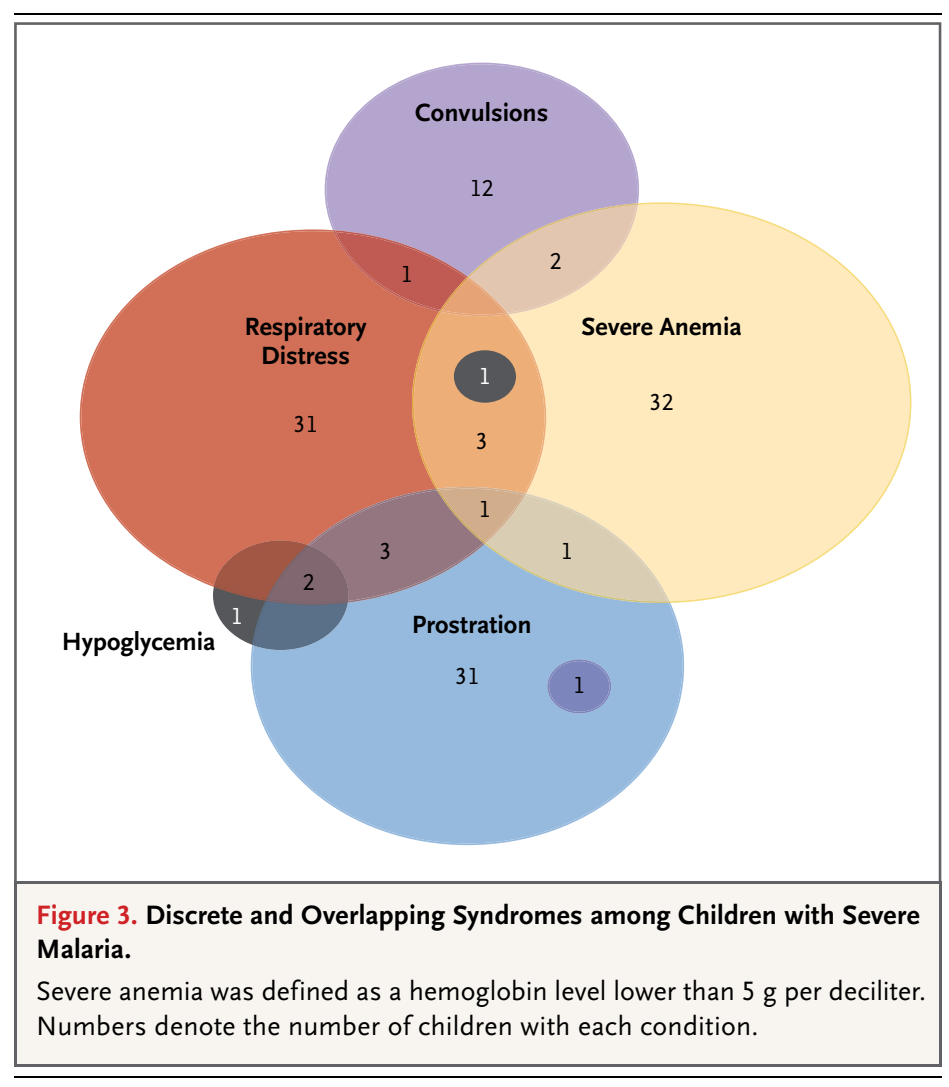

causality: parasite levels are higher during severe episodes, and several risk factors for severe malaria also affect parasite density. Because these factors might modify the risk of severe malaria through their effect on parasite density, interventions designed to reduce parasite density, such as vaccines targeting merozoites, might provide at least partial protection against severe disease. Possibly because responses to multiple variants are required to block erythrocyte infection, ${ }^{27,28}$ early merozoite vaccine candidates did not reduce levels of blood-stage parasites, ${ }^{29-31}$ and their efficacy against severe malaria has therefore not been assessed.

Our findings are in agreement with those of earlier studies showing that sickle cell trait, ${ }^{24,32}$ the low-transmission season, ${ }^{33}$ and bed-net use $^{34,35}$ are associated with reduced parasite density. A new finding is that placental malaria at the time of second or subsequent delivery increases the risk of severe malaria in a woman's offspring, which is similar to the reported effect of placental malaria in such women on the overall risk of infection ${ }^{23}$ and on the risk of 


\begin{tabular}{|c|c|c|c|c|}
\hline \multirow[t]{2}{*}{ Factor } & \multicolumn{2}{|c|}{ Severe Malaria* } & \multicolumn{2}{|c|}{ Parasite Density'̀ } \\
\hline & $\begin{array}{l}\text { Hazard Ratio } \\
(95 \% \mathrm{CI})\end{array}$ & P Value & $\begin{array}{l}\text { Coefficient } \\
(95 \% \mathrm{Cl})\end{array}$ & P Value \\
\hline Sickle cell genotype hemoglobin AS vs. AA & $\begin{array}{c}0.59 \\
(0.30 \text { to } 1.14)\end{array}$ & 0.11 & $\begin{array}{c}-0.46 \\
(-0.68 \text { to }-0.23)\end{array}$ & $<0.001$ \\
\hline Bed-net use vs. no bed-net use & $\begin{array}{c}0.48 \\
(0.32 \text { to } 0.73)\end{array}$ & 0.001 & $\begin{array}{c}-0.52 \\
(-0.71 \text { to }-0.33)\end{array}$ & $<0.001$ \\
\hline Male sex & $\begin{array}{c}0.72 \\
(0.48 \text { to } 1.06)\end{array}$ & 0.10 & $\begin{array}{c}0.03 \\
(-0.14 \text { to }-0.20)\end{array}$ & 0.70 \\
\hline \multicolumn{5}{|l|}{ Village of residence } \\
\hline Magila & Reference & - & Reference & - \\
\hline Bwembwera & $\begin{array}{c}0.87 \\
(0.50 \text { to } 1.52)\end{array}$ & 0.63 & $\begin{array}{c}0.16 \\
(-0.08 \text { to } 0.40)\end{array}$ & 0.20 \\
\hline Mkanyageni & $\begin{array}{c}0.47 \\
(0.25 \text { to } 0.87)\end{array}$ & 0.02 & $\begin{array}{c}0.25 \\
(0.02 \text { to } 0.48)\end{array}$ & 0.04 \\
\hline Muheza township & $\begin{array}{c}0.39 \\
(0.23 \text { to } 0.66)\end{array}$ & $<0.001$ & $\begin{array}{c}-0.10 \\
(-0.34 \text { to } 0.14)\end{array}$ & 0.42 \\
\hline $\begin{array}{l}\text { High-transmission season vs. low-transmission } \\
\text { season }\end{array}$ & $\begin{array}{c}1.49 \\
(1.00 \text { to } 2.22)\end{array}$ & 0.05 & $\begin{array}{c}0.31 \\
(0.19 \text { to } 0.43)\end{array}$ & $<0.001$ \\
\hline \multicolumn{5}{|l|}{ Maternal parity and placental malaria status } \\
\hline First delivery, no placental malaria & Reference & - & Reference & - \\
\hline $\begin{array}{l}\text { Second or subsequent delivery, no placental } \\
\text { malaria }\end{array}$ & $\begin{array}{c}1.11 \\
(0.66 \text { to } 1.86)\end{array}$ & 0.70 & $\begin{array}{c}0.02 \\
(-0.19 \text { to } 0.24)\end{array}$ & 0.84 \\
\hline First delivery, placental malaria & $\begin{array}{c}1.12 \\
(0.44 \text { to } 2.82)\end{array}$ & 0.81 & $\begin{array}{c}0.16 \\
(-0.20 \text { to } 0.52)\end{array}$ & 0.38 \\
\hline Second or subsequent delivery, placental malaria & $\begin{array}{c}1.86 \\
(0.92 \text { to } 3.77)\end{array}$ & 0.08 & $\begin{array}{c}0.30 \\
(0.01 \text { to } 0.59)\end{array}$ & 0.04 \\
\hline
\end{tabular}

* A Cox multivariate model was used to assess the time to the first episode of severe malaria.

$\dagger$ A multivariate generalized-estimating-equation model was used to assess parasite density $\left(\log _{10}\right.$ number of parasites per 200 white cells) during infection.

clinical malaria ${ }^{36}$ in their offspring. The mechanism behind this observation is unknown, but it suggests that in utero exposure might influence immune responses to malaria parasites in early childhood.

Despite its association with disease, parasite burden alone does not completely explain severe malaria. Most children had high-density infection with only mild symptoms before the episode of severe malaria ( 21 of 102 children), after the episode (55 of 102), or both, and most children (67 of 102), regardless of whether they ever had a high-density infection, did not have their severe malaria episode at the time of their highest-density infection. Parasite sequestration complicates the quantification of parasite density in blood smears; however, analysis of PfHRP-2 levels, which are believed to be a better measure of total parasite mass, yielded the same conclusions as our blood-smear data.
We found that naturally acquired resistance to severe malaria was not accompanied by improved control of parasite density. Although the incidence of severe malaria decreases significantly after the first year of life, children continue to have high-density infections. Mean parasite densities do not differ significantly during infections occurring before and those occurring after severe malaria episodes. An understanding of parasite and host factors other than parasite burden that are associated with severe malaria might lead to identification of targets for new vaccines. For example, P. falciparum erythrocyte membrane protein 1 (PfEMP1), a family of highly variant proteins found on the surface of infected erythrocytes, has been linked to severe disease ${ }^{37,38}$ and might be targeted by vaccines.

We observed that severe malarial anemia was usually caused by an abrupt decrease in the hemoglobin concentration, often exceeding $3 \mathrm{~g}$ per 
deciliter. Severe malarial anemia in children is commonly believed to develop as a chronic process, but the pattern we observed indicated a substantial contribution of the acute infection to hemoglobin loss..$^{39,40}$ These two distinct dynamics of hemoglobin decrease could represent different pathologic processes. ${ }^{39}$

Our study provides direct evidence that severe malaria is unlikely to recur but that it commonly occurs after previous infections, including high-density infections. The immunity to severe malaria is not related to improved control of parasite density. The epidemiologic evidence suggests that naturally acquired immunity targets a conserved feature of the various severe-malaria syndromes, such as parasite virulence or host inflammation. An understanding of the natural history of P. falciparum infection and disease can guide mechanistic studies of the pathogenesis of and immunity to severe malaria, which in turn can lead to the development of new therapeutics.

Supported by the Intramural Research Program of the National Institute of Allergy and Infectious Diseases (NIAID), National Institutes of Health (NIH), and by grants from the NIAID extramural program (R01AI52059), the Fogarty International Center (D43 TW005509) of the NIH, and the Foundation for the NIH through the Grand Challenges in Global Health Initiative, which is funded by the Bill and Melinda Gates Foundation (1364).

Disclosure forms provided by the authors are available with the full text of this article at NEJM.org.

We thank the mothers and their children in the Muheza District for participating in the study; Theonest Mutabingwa for leading the clinical team that managed the care and data collection for study participants; Kun-Lin Lee for performing the PfHRP-2 assays; Wonjong Moon, Bess Sorensen, and Melissa Bolla for database management; Dr. Michael Fay for advice on statistical analyses; and Prof. Alassane Dicko for commenting on an earlier version of the manuscript.

\section{REFERENCES}

1. World malaria report. Geneva: World Health Organization, 2012.

2. Greenwood BM, Bradley AK, Greenwood AM, et al. Mortality and morbidity from malaria among children in a rural area of The Gambia, West Africa. Trans R Soc Trop Med Hyg 1987;81:478-86.

3. Greenwood B, Marsh K, Snow R. Why do some African children develop severe malaria? Parasitol Today 1991;7:277-81

4. Mackintosh CL, Beeson JG, Marsh K. Clinical features and pathogenesis of severe malaria. Trends Parasitol 2004;20: 597-603.

5. Doolan DL, Dobaño C, Baird JK. Acquired immunity to malaria. Clin Micro biol Rev 2009;22:13-36.

6. Gupta S, Snow RW, Donnelly CA, Marsh K, Newbold C. Immunity to noncerebral severe malaria is acquired after one or two infections. Nat Med 1999;5: 340-3.

7. McGregor IA. The passive transfer of human malarial immunity. Am J Trop Med Hyg 1964;3:Suppl:237-9.

8. Ellman R, Maxwell C, Finch R, Shayo D. Malaria and anaemia at different altitudes in the Muheza district of Tanzania: childhood morbidity in relation to level of exposure to infection. Ann Trop Med Parasitol 1998;92:741-53.

9. Mtove G, Amos B, Nadjm B, et al. Decreasing incidence of severe malaria and community-acquired bacteraemia among hospitalized children in Muheza, northeastern Tanzania, 2006-2010. Malar J 2011; 10:320.

10. Noedl H, Bronnert J, Yingyuen K, Attlmayr B, Kollaritsch H, Fukuda M. Simple histidine-rich protein 2 double-site sandwich enzyme-linked immunosorbent assay for use in malaria drug sensitivity testing. Antimicrob Agents Chemother 2005;49:3575-7.

11. Severe falciparum malaria: World
Health Organization, Communicable Diseases Cluster. Trans R Soc Trop Med Hyg 2000;94:Suppl 1:S1-S90.

12. Nelson W. Graphical analysis of system repair data. J Qual Technol 1988;20: 24-35.

13. Piel FB, Patil AP, Howes RE, et al. Global distribution of the sickle cell gene and geographical confirmation of the malaria hypothesis. Nat Commun 2010; 1:104.

14. Wambua S, Mwangi TW, Kortok M, et al. The effect of alpha+-thalassaemia on the incidence of malaria and other diseases in children living on the coast of Kenya. PLoS Med 2006;3(5):e158.

15. Salum FM, Wilkes TJ, Kivumbi K, Curtis CF. Mortality of under-fives in a rural area of holoendemic malaria transmission. Acta Trop 1994;58:29-34.

16. Snow RW, Korenromp EL, Gouws E. Pediatric mortality in Africa: Plasmodium falciparum malaria as a cause or risk? Am J Trop Med Hyg 2004;71:Suppl:16-24.

17. Rowe AK, Rowe SY, Snow RW, et al. The burden of malaria mortality among African children in the year 2000. Int J Epidemiol 2006;35:691-704.

18. Nevill CG, Some ES, Mung'ala VO, et al. Insecticide-treated bednets reduce mortality and severe morbidity from malaria among children on the Kenyan coast. Trop Med Int Health 1996;1:139-46.

19. Snow RW, Schellenberg JR, Peshu N, et al. Periodicity and space-time clustering of severe childhood malaria on the coast of Kenya. Trans R Soc Trop Med Hyg 1993;87:386-90.

20. Snow RW, Omumbo JA, Lowe B, et al. Relation between severe malaria morbidity in children and level of Plasmodium falciparum transmission in Africa. Lancet 1997;349:1650-4.

21. Marsh K, Forster D, Waruiru C, et al. Indicators of life-threatening malaria in
African children. N Engl J Med 1995;332: 1399-404.

22. Reyburn H, Mbatia R, Drakeley C, et al. Association of transmission intensity and age with clinical manifestations and case fatality of severe Plasmodium falciparum malaria. JAMA 2005;293:1461-70. 23. Mutabingwa TK, Bolla MC, Li JL, et al. Maternal malaria and gravidity interact to modify infant susceptibility to malaria. PLoS Med 2005;2(12):e407.

24. Aidoo M, Terlouw DJ, Kolczak MS, et al. Protective effects of the sickle cell gene against malaria morbidity and mortality. Lancet 2002;359:1311-2.

25. Erunkulu OA, Hill AV, Kwiatkowski DP, et al. Severe malaria in Gambian children is not due to lack of previous exposure to malaria. Clin Exp Immunol 1992; 89:296-300.

26. O'Meara WP, Bejon P, Mwangi TW, et al. Effect of a fall in malaria transmission on morbidity and mortality in Kilifi, Kenya. Lancet 2008;372:1555-62.

27. Richards JS, Beeson JG. The future for blood-stage vaccines against malaria. Immunol Cell Biol 2009;87:377-90.

28. Thera MA, Doumbo OK, Coulibaly D, et al. A field trial to assess a blood-stage malaria vaccine. N Engl J Med 2011;365: 1004-13.

29. Ogutu BR, Apollo OJ, McKinney D, et al. Blood stage malaria vaccine eliciting high antigen-specific antibody concentrations confers no protection to young children in Western Kenya. PLoS One 2009; 4(3):e4708.

30. Malkin E, Long CA, Stowers AW, et al. Phase 1 study of two merozoite surface protein 1 (MSP1(42)) vaccines for Plasmodium falciparum malaria. PLoS Clin Trials 2007;2(4):e12.

31. Sagara I, Dicko A, Ellis RD, et al. A randomized controlled phase 2 trial of the blood stage AMA1-C1/Alhydrogel malaria 
vaccine in children in Mali. Vaccine 2009; 27:3090-8.

32. Williams TN, Mwangi TW, Wambua $\mathrm{S}$, et al. Sickle cell trait and the risk of Plasmodium falciparum malaria and other childhood diseases. J Infect Dis 2005;192: 178-86.

33. Molineaux L, Gramiccia G. The Garki project: research on the epidemiology and control of malaria in the Sudan savanna of West Africa. Geneva: World Health Organization, 1980

34. Procacci PG, Lamizana L, Kumlien S, Habluetzel A, Rotigliano G. Permethrin- impregnated curtains in malaria control. Trans R Soc Trop Med Hyg 1991;85:181-5.

35. Snow RW, Rowan KM, Lindsay SW Greenwood BM. A trial of bed nets (mosquito nets) as a malaria control strategy in a rural area of The Gambia, West Africa. Trans R Soc Trop Med Hyg 1988;82:212-5.

36. Schwarz NG, Adegnika AA, Breitling LP, et al. Placental malaria increases malaria risk in the first 30 months of life. Clin Infect Dis 2008;47:1017-25.

37. Warimwe GM, Fegan G, Musyoki JN, et al. Prognostic indicators of life-threatening malaria are associated with distinct para- site variant antigen profiles. Sci Transl Med;4:129ra45.

38. Warimwe GM, Keane TM, Fegan G, et al. Plasmodium falciparum var gene expression is modified by host immunity. Proc Natl Acad Sci U S A 2009;106: 21801-6.

39. Weatherall DJ, Abdalla S. The anaemia of Plasmodium falciparum malaria. Br Med Bull 1982;38:147-51.

40. Casals-Pascual C, Roberts DJ. Severe malarial anaemia. Curr Mol Med 2006;6: 155-68.

Copyright (C) 2014 Massachusetts Medical Society. 